\title{
Defective amalgam restorations - repair or replace?
}

\section{Abstracted from}

Sharif Mo, Merry A, Catleugh M et al.

Replacement versus repair of defective restorations in adults: amalgam. Cochrane Database Syst Rev 2014; 2: Art. No.: CD005970. DOI: 10.1002/14651858.CD005970.pub3.

Address for correspondence: Cochrane Oral Health Group, School of Dentistry, The University of Manchester, Coupland 3 Building, Oxford Road, Manchester, UK. M13 9PL E-mail: cohg@manchester.ac.uk

\section{Question: Should we repair or replace defective amalgam restorations?}

Data sources The Cochrane Oral Health Group's Trials Register, the Cochrane Central Register of Controlled Trials (CENTRAL), Medline, Embase, BIOSIS via Web of Knowledge, Web of Science and Opengrey databases were searched. In addition researchers and experts in the field were contacted to trace unpublished or ongoing studies. No restrictions were placed on the language or date of publication. Study selection Randomised controlled trials (including split-mouth studies), involving replacement and repair of amalgam restorations in adults with a defective molar restoration in permanent molar or premolar teeth were to be considered.

Data extraction and synthesis Two review authors independently assessed titles and abstracts for each article identified by the searches in order to decide whether the article was likely to be relevant. Full papers were obtained for relevant articles and both review authors studied these. The Cochrane Collaboration statistical guidelines were to be followed for data synthesis.

Results The search strategy retrieved 201 potentially eligible studies after de-duplication. After examination of the titles and abstracts, full texts of the relevant studies were retrieved but none of these met the inclusion criteria of the review.

Conclusions There are no published randomised controlled trials relevant to this review question. There is therefore a need for methodologically sound randomised controlled trials that are reported according to the Consolidated Standards of Reporting Trials (CONSORT) statement (www.consort-statement.org/). Further research also needs to explore qualitatively the views of patients on repairing versus replacement and investigate themes around pain, distress and anxiety, time and costs.

This paper is based on a Cochrane Review published in the Cochrane Library 2014, issue 2 (see www.thecochranelibrary.com for information). Cochrane Reviews are regularly updated as new evidence emerges and in response to feedback, and the Cochrane Library should be consulted for the most recent version of the review.

\section{Commentary}

The most common reasons for replacing amalgam restorations are secondary caries and tooth restoration fractures (>70\%). ${ }^{1,2}$ Amalgam restoration defects are common, but are often misdiagnosed as secondary caries. ${ }^{3,4}$

Most dentists have been trained to completely remove the defective restoration and replace it with a new one. However, the concept of conservatively repairing only the restoration's caries or defect is now gaining momentum. ${ }^{5}$ This treatment approach is not only effective, but beneficial to the patient as it minimises the unnecessary removal of healthy tooth structure and reduces the risk of irreversible pulpal irritation. ${ }^{6}$

The clinical question Sharif et al. are trying to answer through this systematic review is relevant and appropriate in this age of evidence-based dental practice. ${ }^{7}$ Unfortunately, the strict Cochrane Collaboration criteria, to include only randomised controlled trials with minimal risk of bias, led to an 'empty review'.

It is unlikely that any future study would ever meet the strict inclusion criteria set by this review protocol because it would be unethical to randomly assign a patient with an obvious carious lesion around an amalgam restoration to the 'no treatment' control group.

Sharif et al. made the following suggestion to guide clinical decision-making:

"In the absence of any high quality evidence, clinicians should base their decisions on clinical experience [anecdotal evidence], individual circumstances and in conjunction with patients' preferences where appropriate."

Quality of evidence can range from clear and convincing to invalid and inadmissible. In the hierarchy of clinical evidence, other types of clinical research designs are considered better quality evidence than clinical anecdotes.

Two long-term prospective cohort studies were cited by this systematic review, however they were excluded because participants were not randomised into a control group. Nevertheless, these studies offer a higher level of clinical evidence than 'clinical experience', and are briefly considered here.

The first study, by Gordan et al., followed 50 patients from Florida with a total of 113 defective amalgam restorations over seven years. ${ }^{8}$ After being assigned to different treatment groups, the quality of the restorations were evaluated based on the US Public Health Service (USPHS) criteria. ${ }^{9}$ 
Although a high number of patients were lost to follow-up, the results show that repair of defective amalgam restorations is a good alternative to replacement; only two of the $21(9.5 \%)$ repaired or refurbished amalgam restorations were considered clinically unacceptable as compared to the two of five (40\%) amalgam replacements that were diagnosed as unacceptable and needed further treatment.

The same research group conducted a similar study in Chile on 52 patients with a total of 160 defective amalgam restorations. ${ }^{10}$ A lower number of restorations were reported as lost-to-follow-up after five years.

The authors found that repair or refurbishing defective restorations was as effective as the total replacement of restorations in most circumstances, with only $6.3 \%$ of all the restorations in the experimental group requiring further treatment during the study. In this study, only the no-treatment group showed any signs of secondary caries throughout the five years' study duration.

Despite the limitations of both of these studies (for example, the low sample size, the high loss to follow-up and the lack of ideal randomisation and study blinding) both studies had the same conclusion: when it is feasible, repairing a defective amalgam restoration may be more beneficial and at a lower cost to the patient than the complete replacement of the defective amalgam restoration.

Ben Balevi

University of British Columbia, Vancouver, Canada

1. Opdam NJ, Bronkhorst EM, Roeters JM, Loomans BA. A retrospective clinical study on longevity of posterior composite and amalgam restorations. Dent Mater 2007; 23 2-8

2. Forss $\mathrm{H}$, Widström $\mathrm{E}$. Reasons for restorative therapy and the longevity of restorations in adults. Acta Odontol Scand 2004; 62: 82-86.

3. Pimenta LA, Navarro MF, Consolaro A. Secondary caries around amalgam restorations. J Prosthet Dent 1995; 74: 219-222.

4. Kidd EA, Joyston-Bechal S, Beighton D. Marginal ditching and staining as a predictor of secondary caries around amalgam restorations: a clinical and microbiological study. J Dent Res 1995; 74: 1206-1211.

5. Hickel R, Brüshaver K, llie N. Repair of restorations - criteria for decision-making and clinical recommendations. Dent Mater 2013; 29: 28-50.

6. Opdam NJ, Bronkhorst EM, Loomans BA, Huysmans MC. Longevity of repaired restorations: a practice-based study. J Dent 2012; 40: 829-835.

7. Sharif MO, Merry A, Catleugh M, et al. Replacement versus repair of defective restorations in adults: amalgam. Cochrane Database Syst Rev 2014; 2: Art. No. CD005970. DOI: 10.1002/14651858.CD005970.

8. Gordan V, Riley JL 3rd, Blaser PK, Mondragon E, Garvan CW, Mjör IA. Alternative treatments to replacement of defective amalgam restorations: results of a seven-year clinical study. J Am Dent Assoc 2011; 142: 842-849.

9. Cvar JF, Ryge G. Reprint of criteria for the clinical evaluation of dental restorative materials. 1971. Clin Oral Investig 2005; 9: 215-232.

10. Martin J, Fernandez E, Estay J, Gordan VV, Mjör IA, Moncada G. Management of Class I and Class II Amalgam Restorations with Localized Defects: Five-Year Results. Int J Dent 2013; 2013: 450260. doi: 10.1155/2013/450260.

Evidence-Based Dentistry (2014), 15, 54-55. doi: 10.1038/sj.ebd.6401028 